\title{
La résidence confrontée à la recherche en arts numériques
}

\author{
Stéphane Bellin \\ Avignon Université, \\ Centre Norbert Elias, Équipe Culture \& Communication
}

Les arts numériques résultent de l'utilisation des technologies numériques à des fins de création (Couchot \& Hillaire, 2003). Cet attribut a facilité leur disposition à se situer au croisement de la recherche artistique et de la recherche technologique (Dallet, 2012). Aujourd'hui, en France, la recherche en arts numériques regroupe des conceptions et des pratiques hétérogènes (Bellin, 2017). Elles changent considérablement selon les institutions qui l'organisent : des entreprises, des universités, des écoles supérieures d'art, des centres d'art ou des lieux hybrides. Dans cet article, elle est envisagée comme relevant simultanément de l'expérimentation artistique et de l'innovation technologique. La première constitue le processus créatif, y compris ses erreurs et ses tâtonnements, comme un acte de recherche. La deuxième aboutit à l'émergence de dispositifs techniques originaux. Leur rapprochement suscité par les arts numériques repose sur deux postulats : "l'art permettrait [...] de penser et d'anticiper des usages technologiques innovants [...] et d'autre part, il y aurait aussi l'idée que la recherche artistique peut donner lieu à des développements technologiques inédits " (Fourmentraux, 2011 : 26-27). En assumant 
pleinement la dimension technique de l'art, la recherche en arts numériques n'est pas repliée seulement sur l'étude du fait artistique. Au contraire, elle expérimente par un processus créatif une problématique commune à l'art et à la technique.

Le sociologue Jean-Paul Fourmentraux atteste que la recherche en arts numériques transforme les modalités du travail artistique. Ce dernier s'y adapte pour répondre à " une pluralité d'enjeux " et de "logiques de production" - artistique, technologique et économique (ibid. : 35). Inscrit dans une " organisation par projet " (ibid. : 37), il devient davantage interdisciplinaire et collectif (ibid. : 103). Il ne se focalise plus exclusivement sur la production d'œuvres considérées comme des objets finaux, mais "favorise l'expérimentation et l'essai-erreur " (ibid. : 34). Il en résulte des " œuvres frontières [qui] tendent à devenir plus flexibles que ne le prévoient leurs définitions habituelles, afin de s'adapter aux besoins et aux nécessités spécifiques des différents acteurs "(ibid. : 103). Plus largement, les résultats de la recherche en arts numériques sont pluriels et fragmentés : "les "créations artistiques", qui mènent vers la réalisation d'une ouvre, d'un dispositif ou d'une installation artistique ; les "découvertes technologiques", qui impliquent le développement de logiciels ou d'outils novateurs ; les "contributions théoriques", qui poursuivent une perspective analytique et critique d'accumulation de connaissances " (Bianchini, Fourmentraux \& Mahé, 2009 : 138). Face à ces constats, Fourmentraux affirme que l'un des principaux enjeux pour les acteurs de la recherche en arts numériques est de définir " de nouveaux modèles organisationnels et de gestion [...] susceptible[s] de promouvoir des valorisations tout à la fois esthétiques, scientifiques et industrielles " (ibid. : 38). Si ces modèles ne sont pas encore fixés, certains de ces acteurs se sont emparés des résidences pour atteindre cet objectif. Le présent article propose de les examiner. Il tente de répondre à cette problématique : comment la résidence artistique prend-t-elle en compte la démultiplication des enjeux de production et de communication de la recherche en arts numériques? 
Cet article s'appuie principalement sur l'étude des résidences organisées par l'association montpelliéraine Kawenga Territoires numériques ${ }^{1}$. Elle s'est déroulée sur une période de deux ans en 2011 et en 2012. Cette étude correspond à une recherche doctorale qui, dans le cadre d'une convention $\mathrm{CIFRE}^{2}$, m'a conduit à être embauché pendant deux ans pour y interroger les potentialités de la documentation à communiquer les activités accomplies par les artistes accueillis en résidences (Bellin, 2017). Un protocole documentaire a été mis au point. Il débute par des entretiens qualitatifs semi-directifs menés auprès de l'artiste et ses collaborateurs. Il s'agit de leur faire adopter le recul nécessaire pour réfléchir à leurs pratiques et les analyser. Les guides d'entretiens sont élaborés à partir de l'examen des dossiers de financements déposés par les artistes. Ils permettent de les interroger sur l'émergence du projet artistique, sur ses principaux concepts et idées, sur les principaux médias et médiums utilisés, sur les outils et les composantes technologiques sur lesquels il repose. Un deuxième entretien est effectué en fin de résidence pour les questionner sur le travail directement effectué pendant la résidence, notamment sur le caractère versatile et empirique du processus créatif. En parallèle, des observations participantes sont réalisées pendant les phases de l'expérimentation artistique et des captations audio-visuelles sont effectuées. Les actions de médiation sont elles aussi enregistrées. Une recherche documentaire sur l'équipe artistique et leurs œuvres complète le travail de documentation. Tous les documents ainsi produits ou recueillis sont exploités selon les techniques de l'analyse documentaire pour rédiger et mettre en forme le cahier de résidence, une publication dont l'objectif est de rendre compte et de transmettre un processus créatif expérimental et des connaissances extérieures à l'œuvre d'art. Pendant deux ans, cinq résidences

1. L'association a cessé ses activités en juillet 2013, pour des raisons de financements.

2. Convention industrielle de formation par la recherche. Ce dispositif financier doctoral subventionne toute entreprise ou association de droit français qui embauche un doctorant pour le placer dans une situation de recherche dans ses locaux et par rapport à ses activités en collaboration avec un laboratoire universitaire public. 
ont ainsi été documentées ${ }^{3}$. Au-delà de ce travail, la rédaction d'un journal de bord m'a permis de me détacher de cette pratique de documentation des résidences pour mieux déployer mon action de chercheur. Cet outil méthodologique m'est apparu comme le moyen de conduire ma démarche de recherche par l'action, ce qui m'a positionné de manière simultanée dans la pratique et dans l'analyse. Pendant deux ans, en tenant ce journal de bord quotidiennement, j'ai documenté mon travail de praticien, par des prises de notes de réunions ou issues de mes observations, par des réflexions personnelles sur les manières dont j'ai pensé et construit le dispositif documentaire ou encore sur les difficultés rencontrées. Ces écrits constituent en quelque sorte un retour sur le travail entrepris. Pour cet article, d'autres documents produits en interne par l'équipe de Kawenga ont été exploités, par exemple des comptes rendus de réunion ou des bilans d'activités. L'ensemble de ce corpus documentaire a été examiné selon une grille commune articulée autour de trois axes thématiques généraux : l'organisation, les résultats produits et les formes de médiation des résidences. Pour chacun de ces thèmes, l'objectif a été d'en déterminer les enjeux afin de les comparer à ceux de la recherche en arts numériques.

Pour éviter une étude trop circonscrite à un seul terrain, l'analyse des résidences de Kawenga a été complétée avec celles qui ont rythmé la production de l'œuvre intitulée Tropique de l'artiste Étienne Rey ${ }^{4}$ dans le cadre du RAN (Réseau arts numériques). En effet, ce projet a été désigné en juin 2011, avec deux autres, comme lauréats du premier appel à projets initié par le RAN. Ce réseau international associe vingt-six structures qui collaborent à des projets participant à la convergence des arts, des technologies

\footnotetext{
3. Chaque résidence concerne un projet artistique précis : S.U.N de la chorégraphe Young-Go Nam, Projet Liban / Ahlan wa sahlan de la compagnie Arcinolether, Golem de l'artiste Thomas Tudoux, L'Iconopathe de la photographe d'Isabelle Massu et Macadam Instinct du chorégraphe Yann Lheureux dans le cadre du projet Scène(s) Numérique(s).

4. Celle-ci adopte la forme d'une installation immersive à travers laquelle le visiteur fait l'expérience d'un espace embrumé, sculpté par la lumière et le son. Cf. Rey (Étienne). (2013). "Tropique ». Etienne Rey. Atelier des ondes parallèles. Récupéré le 15 août 2017 de http://ondesparalleles.org/projets/tropique-7/
} 
numériques et des sciences. Or, pour atteindre ce dessein, elles ont adopté la résidence comme modèle organisationnel. Ce deuxième volet de l'étude n'a pas requis le même engagement participatif de ma part. Les résidences du projet Tropique ont été étudiées rétrospectivement à travers l'examen des discours véhiculés par une multitude de documents. Ces derniers ont été produits par les différentes institutions afin de communiquer sur les résidences qu'elles organisent. La grille d'analyse thématique utilisée est la même que celle mobilisée pour l'étude des résidences de Kawenga.

Le présent article s'organise en cinq parties. La première évoque le positionnement de l'association Kawenga dans la recherche en arts numériques lors de sa transformation en médialab artistique. La deuxième expose la conséquence sur les résidences, qui se traduit par le retrait de l'œuvre au profit de l'expérimentation artistique et technologique. La troisième aborde les effets sur la dimension communicationnelle de ces mêmes résidences, cristallisée autour des actions de médiation, tandis que la quatrième se focalise sur la médiation documentaire et sa capacité à créer une communication différée dans le temps. La cinquième partie tente de mesurer la récurrence de ce modèle résidentiel à partir d'un autre contexte, celui du RAN.

\section{L'association Kawenga : l'instauration d'un médialab artistique}

Depuis sa création en 1999, Kawenga a régulièrement évolué. Au départ, l'association était intégrée dans le réseau des Espaces Culture Multimédia (ECM) (Théobalt, 2001). Elle proposait un accès à des ressources multimédia sur des contenus artistiques et culturels, organisait des ateliers d'appropriation des outils et des logiciels numériques à des fins de création, et invitait des conférenciers pour mener des débats sur les enjeux de la culture numérique émergente. En septembre 2008, commence une réorientation du projet associatif. Dès son arrivée, la nouvelle directrice a renforcé la présence et le rôle des artistes au sein de la structure. 
Cette volonté reposait sur l'idée que l'appropriation des arts numériques par des publics amateurs serait stimulée à leur contact. Aussi, un "pôle création " a-t-il été créé. Celui-ci s'est matérialisé, dans de nouveaux locaux, par une pièce destinée à accueillir des artistes en résidences. Un chargé de production a été embauché pour en gérer l'organisation. Ce "pôle création " est devenu un axe important du développement de Kawenga, car il a joué le rôle de levier pour inciter les artistes à investir le lieu et à participer à la réalisation d'interventions auprès des publics. Dans ce sens, il a contribué à alimenter les différentes modalités publiques et à structurer les autres pôles de l'association'.

En 2011, Kawenga devient un médialab artistique. Le concept de médialab désigne des lieux hybrides, dans lesquels se croisent et se réunissent des acteurs de l'innovation numérique dans une démarche créative, collaborative et interdisciplinaire, voire transdisciplinaire (Lenoble, 2011 : 6). Les médialabs se sont développés à partir des années 2000 en s'inspirant d'un modèle précurseur, celui du MIT Media Lab ${ }^{6}$, créé en 1985 aux États-Unis. Ils participent à la mise en place d'une innovation de réseau (Bianchini, Fourmentraux \& Mahé, 2009 : 142), c'est-à-dire qu'ils ambitionnent de fédérer dans une même structure et autour de projets communs tous les maillons de l'innovation numérique dans un territoire donné. La prise en compte de ces derniers révèle également la volonté de contribuer au développement de l'innovation ascendante, également nommée l'innovation par l'usage (Cardon, 2005). Elle se caractérise par l'idée que l'innovation numérique émane, non plus seulement des laboratoires de recherche et de développement des universités et des entreprises, mais aussi des usagers eux-mêmes. Dès lors, la mutation de Kawenga en médialab traduit le désir de prendre en compte le rôle des usagers dans l'innovation ascendante, qu'il s'agisse des professionnels-amateurs (Flichy, 2010 : 8) ou des artistes (Bianchini,

\footnotetext{
5. En plus du "pôle création ", Kawenga se composait de quatre pôles supplémentaires : le "pôle espace public ", le "pôle activités éducatives ", le " pôle ressources " et le "pôle coopération territoriale".

6. Le MIT Media Lab est un laboratoire du Massachusetts Institute of Technology, dédié aux projets de recherche mêlant design, numérique et technologie.
} 
2009 : 35-36). La stratégie étant de mettre en place au niveau régional d'un médialab artistique, c'est-à-dire un lieu dédié à l'expérimentation des arts et des cultures numériques, Kawenga devient une sorte d'incubateur artistique et culturel autour des technologies numériques au carrefour d'un laboratoire scientifique, d'un centre d'art et d'une entreprise.

\section{Le retrait de l'œuvre au profit de l'expérimentation artistique et technologique}

Inspirée de l'art expérimental (During, Jeanpierre \& Kihm, 2009), Kawenga est alors sollicitée pour activer la recherche en arts numériques. Celle-ci comporte par définition une grande part d'incertitude. Selon le sociologue Pierre-Michel Menger, cette caractéristique est essentielle pour mener un travail artistique innovant : " c'est l'incertitude sur le cours de l'activité et son résultat qui est la condition de l'invention originale, et de l'innovation à plus longue portée " (Menger, 2009 : 11). Il considère également l'expérimentation artistique comme le paroxysme de l'incertitude créative, car elle se "veut purement aléatoire " (ibid. : 677). Afin de stimuler les potentialités d'innovation, la structure se centre non plus sur la production d'une œuvre finalisée, mais davantage vers l'expérimentation du processus créatif. Ce constat rejoint celui formulé par Fourmentraux, déjà cité en introduction, pour qui le travail artistique dans un contexte de recherche en arts numériques ne se focalise plus exclusivement sur la production d'œuvres considérées comme des objets finaux, mais "favorise l'expérimentation et l'essai-erreur" (Fourmentraux, 2011 : 34). Si ce type de recherche fait des résidences un espace potentiellement productif d'œuvre d'art, la priorité est davantage accordée à la démarche de recherche portée par l'artiste. Cependant, les financements sur lesquels s'appuie l'organisation des résidences de Kawenga réclament le plus souvent la réalisation d'une œuvre finalisée, bien que leur obtention dépende en amont d'une évaluation prospective du processus de création. Par exemple, si le projet obtient une aide à 
la production du DICRéAM ${ }^{7}$ ou de la Région LanguedocRoussillon, Kawenga doit trouver des partenaires prêts à diffuser l'œuvre produite pendant la résidence. Il s'agit de répondre aux exigences de résultats et de visibilité des institutions qui subventionnent. Ces financements par projet sont fondés sur une évaluation du " processus créatif et c'est la démarche qui est jugée et qui donne droit à des aides à la production, le plus souvent sous la forme de subventions " (De Vrièse et al., 2011 : 9).

Cette évaluation de la faisabilité des projets requiert l'élaboration d'un dossier de candidature dans lequel les enjeux et les moyens artistiques et techniques sont exposés en détail, ce qui laisse finalement très peu de place aux imprévus et à l'incertitude. Or, l'expérimentation artistique ne peut pas être prédéterminée à l'avance dans tous ses aspects. Au contraire, elle tend à aboutir à une ouvre "dont l'avancement n'obéirait à aucune formule initiale planificatrice, ni à aucune mise en cohérence progressive " (Menger, 2009 : 677-678). En outre, à Kawenga, le travail artistique est souvent évalué à l'issue de la résidence et uniquement à travers l'œuvre produite, et le processus créatif, notamment son caractère expérimental comprenant des hésitations et des erreurs, est ignoré. Paradoxalement, il semble donc que l'expérimentation artistique est légitime seulement quand elle est soumise en amont à une forte prévisibilité et lorsqu'elle est productrice en aval d'œuvres originales de qualité. De surcroît, dans le domaine de la recherche en arts numériques qui mobilise aussi l'expérimentation technologique, le développement de solutions et d'outils techniques ne sont pas pris en compte comme résultats potentiels.

Avant sa transformation en médialab artistique, Kawenga organisait des résidences qualifiées de "résidence mixte " dans le domaine littéraire par la chercheuse Carole Bisenius-Penin (2016 : 14) ou de "résidences de création d'œuvre(s) et d'intervention " par le Centre national des arts plastiques, (CNAP) dans son guide des résidences d'arts visuels en France (CNAP, 2016 : 12). Ce type de résidences

7. Le dispositif national d'aide à la création multimédia (DICRéAM) est administré par le Centre National du Cinéma (CNC). 
se caractérise par une temporalité partagée entre la création d'une œuvre et des actions de médiation auprès de publics, le temps consacré à la première étant plus conséquent que celui dédié aux secondes. Carole Bisenius-Penin évoque des résidences " dite[s] "de création", d'expérimentation, centrée[s] essentiellement sur un projet littéraire dans laquelle l'institution culturelle partenaire demande peu ou pas du tout de contrepartie aux auteurs, le temps étant entièrement dédié au processus de création " (BiseniusPenin, 2016 :14). Dans le domaine des arts plastiques, Olivier Le Falher évoque "un modèle alternatif, visant à garantir l'entière autonomie des artistes au travail " pour lequel " les responsables d'associations ou de centres d'art choisissent de laisser l'artiste libre d'expérimenter et de se confronter seul à un travail d'atelier sans objectif de production ni contrainte de résultats "(Le Falher, 2014 : 85). À Kawenga, le choix a été pris de ne pas s'orienter vers ce type de résidences, en raison du type de subventions reçues. L'ambition de diminuer l'importance accordée à la production des ouvres au profil de l'expérimentation artistique et technologique s'exprime donc dans les actions de médiation proposées aux publics pendant les résidences.

\section{La médiation comme moyen de communication}

Les résidences organisées par Kawenga se prolongent toujours par des actions de médiation, dont l'objectif général est de créer la rencontre avec des publics. Il en en existe plusieurs formes ${ }^{8}$. Pour chacune d'entre elles, l'artiste et ses collaborateurs communiquent aux publics le travail artistique expérimenté pendant la résidence. Jean Davallon relève que dans la médiation " la communication [...] apparaît comme la mise en ouvre d'un élément tiers rendant possible l'échange social, alors même que les univers de la production et de la réception sont a priori par nature disjoints "(Davallon, 2003 : 53). Dans le domaine culturel, il précise qu'elle " vise à faire accéder un public à des œuvres

8. La présentation publique, le workshop, la Convergence et le Cahier de résidence. 
(ou des savoirs) et son action consiste à construire une interface entre ces deux univers étrangers l'un à l'autre dans le but précisément de permettre une appropriation du second par le premier "(ibid. : 38). L'expérimentation du processus créatif est par nature un élément difficile à communiquer. Fortement attachée au travail artistique, elle appartient uniquement à ceux qui la mettent en action, s'exerce dans l'immédiateté du temps réel et apparaît donc comme éphémère. Aussi, les actions de médiation apparaissent-elles comme des dispositifs tiers qui permettent tout de même au public de s'approprier la complexité d'un processus créatif expérimental mené pendant les temps de création, dont il est écarté.

Si les actions de médiations sont différentes les unes des autres, elles se rejoignent dans la volonté de permettre aux publics d'aborder le travail artistique, non plus à partir d'une ouvre finalisée, mais comme un processus créatif expérimental. Dès lors, elles ne se polarisent pas sur la monstration de l'œuvre finalisée, considérée comme le seul aboutissement du processus créatif expérimental. Elles s'organisent plutôt autour d'une multitude de résultats intermédiaires et fragmentés, observés par Jean-Paul Fourmentraux dans d'autres contextes de recherche en arts numériques (Fourmentraux, 2011 : 35). Deux exemples peuvent attester de ce parti pris : la présentation publique et le workshop. La première cherche à susciter une discussion entre le public et les équipes artistiques et techniques autour des expérimentations menées pendant une résidence. Pour cela, elle s'ouvre souvent par la monstration d'un prototype artistique, dont la fonction est de matérialiser l'expérimentation artistique et technologique sous une forme tangible. En règle générale, ce prototype précède une œuvre finalisée qui, elle, n'est presque jamais exposée dans les locaux de Kawenga. Le second, le workshop, adopte la forme d'un atelier. Sa particularité est le rapport qu'il entretient avec la résidence. En effet, il propose une séance de créativité, au cours de laquelle les stagiaires sont eux-mêmes mis en situation d'expérimentation autour du processus créatif exploré par l'artiste et ses collaborateurs. Ainsi, ils échangent et collaborent ensemble en évoquant les connaissances, les techniques et les savoir-faire mobilisés 
pendant la résidence. Sa dimension pratique oriente souvent les discussions sur la technique. Le workshop valorise ainsi le dispositif technique développé et les savoir-faire qui y sont liés.

Dans la circulaire no 2006-01 du 13 janvier 2006, le Ministère de la culture précise que la rencontre avec les publics " ne prend pas obligatoirement la forme d'un spectacle, d'une exposition ou d'une publication " pour une résidence " où la démarche de recherche ou d'expérimentation est dominante " (ministère de la Culture et de la communication, 2006 : 6). Il précise qu'" elle peut recouvrir par exemple la réalisation d'une maquette constituant la première étape d'une démarche de création appelée éventuellement à se poursuivre [et] elle doit alors être présentée comme telle au public "( ibid.). Les résidences de Kawenga s'inscrivent dans cette conception de la résidence d'expérimentation et cette logique communicationnelle.

\section{La médiation documentaire pour une communication différée}

Dans cet article, le concept de médiation ne décrit pas seulement des actions culturelles spécifiques, mais il est aussi utilisé pour sa capacité "à dépasser [...] l'opposition entre information et communication " (Bordeaux \& Caillet, 2013 : 147) de manière à " décrire avec une certaine précision les processus d'information-communication " (Jeanneret, 2009 : 26). Dans cette perspective, les actions de médiation observées à Kawenga contribuent à "transférer de l'information " aux publics sur le processus créatif expérimenté pendant la résidence, de sorte que "la médiation est synonyme d'explication de contenu à des destinataires " (Chaumier, 2018 : 40). Si elles s'exercent essentiellement sous la forme d'un échange oral, elles font l'objet - comme cela a déjà été abordé dans l'introduction - d'enregistrements réalisés par le documentaliste afin de collecter la parole de l'artiste et de ses collaborateurs. Aussi, au-delà d'assurer une médiation du processus créatif par l'artiste en face-à-face d'un public, elles assument une fonction supplémentaire, celle d'alimenter le processus de documentation du travail explo- 
ratoire mené en résidence. Dans cette perspective, les actions de médiation sont l'occasion de produire des documents - des enregistrements sonores - qui renseignent sur l'expérimentation artistique et technologique. Il ne s'agit plus seulement de communiquer de l'information en présentiel aux publics, mais de sauvegarder cette information dans des documents.

D'autres documents sont produits et collectés tout au long de la résidence par le documentaliste, comme des photographies prises par les autres membres de l'équipe de Kawenga ou directement par les artistes. L'objectif est de réunir un ensemble de documents diversifiés exploités pour rédiger le cahier de résidence. Il y identifie les informations utiles à la compréhension du processus créatif expérimenté puis, les reformule sous la forme structurée du cahier de résidence ${ }^{9}$. Celui-ci s'organise autour de huit parties ${ }^{10}$. Les plus centrales, intitulées " conception " et " production ", restituent les réflexions issues de l'expérimentation artistique La première en présente les différents concepts et idées créatifs ainsi que les médias et les composants techniques utilisés et développés. Elle expose également la manière dont ses différents éléments simbriquent les uns aux autres. La seconde se compose de deux points, la description des différentes étapes diexploration du processus créatif et lanalyse de lorganisation et des méthodes de travail testées. Chaque partie est abondamment illustrée par des photographies contextualisées et explicitées par des légendes détaillées. Ainsi, le cahier de résidence offre aux lecteurs une représentation à la fois textuelle et visuelle du déroulement de l'expérimentation artistique et technologique. Elle en constitue un substitut documentaire. Disponible en version papier et en version numérique sur le site internet de Kawenga et via une plate-forme gratuite de partage de documents en ligne, elle constitue l'achèvement communicationnel du processus de documentation.

\footnotetext{
9. Un exemple de cahier peut être consulté sur ce lien : https://www.scribd.com/ document/110866112/Cahier-Residence-S-U-N

10. Les huit parties sont les suivantes : l'identité du projet, les protagonistes, la note d'intention, la conception, la production, la réception, des ressources et les contacts des partenaires.
} 
Le cahier de résidence constitue un objet matériel qualifié de médiateur au sens où il sert d'intermédiaire entre l'expérimentation artistique éphémère et les publics qui cherchent en avoir connaissance au-delà de sa temporalité circonscrite. Cette publication fait en sorte que celle-ci demeure disponible pour une lecture ultérieure libérée de la contrainte temporelle. Dès lors, la documentation préserve dans le temps la potentialité informationnelle de l'expérimentation artistique et technologique pour une communication différée auprès de publics susceptibles d'être intéressés par celle-ci. Cette disposition relève de la médiation documentaire, car la documentation permet ici " de concilier deux choses jusque-là non rassemblées pour établir une communication et un accès à l'information " (Gardiès \& Fabre, 2012 : 45). Il se dessine alors une médiation documentaire, qui ne se limite plus au seul traitement documentaire, mais englobe désormais la production de ressources spécifiques par le documentaliste lui-même (Liquète et al., 2010). La médiation documentaire se rapproche des différentes actions de médiation organisées pendant la résidence par le fait qu'elle communique le travail artistique essentiellement à travers le processus créatif expérimental à des publics qui en sont exclus. En revanche, elle s'en distingue sur trois points. D'abord, elle résulte, non pas de l'artiste, mais du travail du documentaliste. Ensuite, elle transmet l'expérimentation artistique et technologique, non pas à l'oral ou sous des formes matérielles fragmentées et intermédiaires - par exemple, un prototype artistique - mais dans une publication structurée et finalisée aux contenus plus explicites. Enfin, elle offre une situation communicationnelle différée et persistante dans le temps.

\section{Récurrence du modèle résidentiel étudié}

La résidence, dans le domaine de la recherche en arts numériques, ne se limite pas au seul exemple de l'association Kawenga, mais concerne un ensemble plus vaste d'institutions, qui permettent une approche comparative, qui sera ici esquissée. La majeure partie est regroupée au sein 
du $\mathrm{RAN}^{11}$. Initié en 2007, par le Centre des arts d'Enghienles-Bains, ce réseau regroupe trente-sept structures. Si elles agissent toutes dans le domaine des arts numériques, elles relèvent d'une grande diversité : douze d'entre elles sont des centres d'art, dix des festivals, trois des entreprises start-up, sept des collectifs d'artistes, six des laboratoires d'écoles ou d'universités, et quatre des médias. Elles relèvent des secteurs de l'art, de la science ou de l'industrie. Cette pluralité suggère la volonté du RAN de décloisonner ces secteurs dans un espace de collaboration commun. Toutes ces structures tentent de se rapprocher en menant ensemble trois types d'actions : la mise en place d'un guide transversal des arts numériques en ligne, une veille sur les nouveaux usages et métiers de la création numérique afin d'optimiser la formation professionnelle, et l'accompagnement de projets pilotes. Le dernier point consiste à coproduire et à diffuser en réseau des ouvres d'art numérique. Elles sont sélectionnées par un appel à projets international. Pour encourager le décloisonnement de l'art, de la science et de l'industrie, le RAN accorde son soutien aux projets en arts numériques, qui se positionnent dans le domaine de la recherche artistique, scientifique et technique. Le comité de sélection sélectionne donc et labellise les projets jugés les plus ambitieux en termes d'expérimentation artistique et technique. En échange, ils bénéficient d'un accompagnement par un comité, d'une aide en coproduction, d'une résidence itinérante et de plusieurs diffusions. Le RAN utilise la résidence itinérante comme un des dispositifs organisationnels de son réseau. Celle-ci comporte plusieurs sessions de travail, qui sont organisées dans différents lieux. Elle suppose que l'artiste se déplace à plusieurs reprises. Pour chacune des sessions, il bénéficie des ressources propres à chacun des lieux qui l'accueillent, et en contrepartie, lorsque que cela est demandé, il s'engage à participer à l'organisation d'actions de médiation. En général, l'artiste ne développe pas les mêmes aspects de son projet d'une session à l'autre. Chacune est consacrée à un développement spécifique de celui-ci. Pour son œuvre

11. Cf. RAN (2007). RAN Réseau arts numériques. Récupéré le 15 août 2017 de http://www.ran-dan.net/ 
"Tropique ", l'artiste Étienne Rey a bénéficié de huit sessions de travail réparties dans six lieux différents ${ }^{12}$. Selon les sessions et les lieux qui les organisent, l'analyse de cette résidence itinérante montre que sa conception alterne entre plusieurs modèles. Par exemple, au Groupe de Musique expérimentale de Marseille - Centre National de Création Musicale, elle adopte les caractéristiques d'une résidence d'expérimentation entièrement dédiée au processus créatif, c'est-à-dire qu'elle ne propose aucune médiation. Ce modèle y est minoritaire, car il est apparu uniquement dans ce lieu. Pour les autres sessions, elle se rapproche du modèle de la résidence d'expérimentation et de recherche en arts numériques, tel qu'il a été décrit dans le cas de Kawenga. Elle comporte des médiations, qui permettent aux publics d'aborder le travail artistique, non plus à partir d'une ouvre finalisée, mais comme un processus créatif expérimental. Par exemple, au Centre des arts d'Enghienles-Bains, la résidence a abouti à une médiation, qui a pris la forme d'une exposition qualifiée de "work in progress". Cette expression indique que l'exposition montrait une œuvre en cours de production, non terminée. Dans ce sens, il s'agissait d'un prototype artistique. Les visiteurs expérimentaient Tropique dans une vision minimale, c'està-dire avec un seul vidéo projecteur, sans interactivité et sans son. Néanmoins, ils faisaient l'expérience du prototype de manière à percevoir sa nature immersive. Lors des actions de médiation, cette expérience amorçait la rencontre des publics avec l'artiste qui leur expliquait sa démarche artistique expérimentale. De cette façon, les visiteurs étaient amenés à élaborer une réflexion collective autour de celle-ci. Dans cette session de la résidence itinérante, l'œuvre était montrée dans une forme intermédiaire, celle du prototype artistique, et les mises en situations communicationnelles de l'artiste et de ses collaborateurs se concentraient sur la démarche de recherche et le processus créatif.

12. L'œuvre Tropique a bénéficié de plusieurs sessions de résidences au sein de différentes structures : La Friche La Belle de Mai, l'IMéRA - Institut d'études avancées de l'Université d'Aix-Marseille -, le GMEM-CNCM-MARSEILLE - Centre national de création musicale - et le CDA, le Centre des arts d'Enghien-les-Bains. 


\section{Conclusion}

Confrontée à la recherche en arts numériques, la résidence demeure une entité hybride et polymorphe. Si elle se partage toujours entre deux dimensions : la création et de la médiation. Une troisième, dont il convient de tenir compte, est celle de la technologie. En effet, ce type de résidence devient le lieu où le travail artistique et l'innovation technologique se rejoignent sous un aspect expérimental. Les attentes s'en trouvent modifiées. L'impératif de production d'une œuvre finalisée passe au second plan, il arrive même parfois que la résidence s'exempte totalement de tout objectif de production. Cependant, le plus souvent, subsiste la contrainte de résultats. Il s'agit de transmettre des savoirs et des repères sur l'expérimentation artistique et technologique. Aussi, la médiation y demeure un axe fort, car elle est sollicitée comme moyen de communication. Quand elle prend la forme d'actions spécifiques, elle privilégie la communication orale autour de la rencontre directe de l'artiste et de ses collaborateurs avec les publics. Quand elle devient médiation documentaire, elle privilégie la communication différée et persistante dans le temps. Quelle que soit sa forme, la médiation opère à la fois au niveau de la dimension artistique et de la dimension technologique. Elle se manifeste comme un dispositif tiers pour permettre aux publics de s'approprier la complexité d'un processus créatif et technologique expérimental, bien qu'ils en soient écartés, du fait qu'ils n'y participent pas.

Au final, la malléabilité de la résidence en tant que modèle organisationnel, entre en adéquation avec la démultiplication des enjeux de production et de communication induits par la recherche en arts numériques. En effet, en raison de son caractère interdisciplinaire et de son organisation collective, cette dernière engendre des résultats divers et fragmentés. Leur valorisation se complexifie dans la mesure où ils ne sont pas réunis dans un seul objet communicationnel et qu'ils concernent sphères sociales différentes, séparées par domaine de compétences. Or, les actions de médiation contournent cette difficulté en permettant de valoriser cha- 
cun de ces résultats composites par la diversité des situations communicationnelles qu'elles offrent. Néanmoins, il conviendrait de mener une étude spécifique pour identifier les publics qui en bénéficient. Quelles sont les motivations qui les poussent à y participer ? À qui profite la médiation documentaire? L'analyse de ces publics corrobore-t-elle la volonté affichée de décloisonner les milieux de l'art, de la science et de l'industrie?

Manuscrit reçu le 1er septembre 2017

Version révisée reçue le 26 juin 2018

Article accepté pour publication le 7 septembre 2018

\section{Bibliographie}

Bellin (Stéphane). 2014. "Documenter et communiquer la recherche en arts numériques par la mise en situation dialogale de l'artiste contemporain ». Muséologies. Les cabiers d'études supérieures, 7(1), p. 210-223.

Bellin (Stéphane). 2017. Communiquer la recherche en arts numériques. Une approche info-communicationnelle de la documentation. Thèse en sciences de l'information et de la communication / sous la direction de Cécile Tardy et Joanne Lalonde. Avignon Université et Université du Québec à Montréal.

Bianchini (Samuel), Fourmentraux (Jean-Paul) \& Mahé (Emmanuel). 2009. "Création et Recherche \& Développement ", p. 136-142 in Valeurs croisées, Les Ateliers de Rennes -Biennale d'art contemporain / sous la direction de Raphaëlle Jeune. Dijon : Les Presses du Réel.

Bianchini (Samuel). 2009. "Recherche \& création ", p. 18-43 in Recherche \& Création. Art, Technologie, pédagogie, innovation / sous la direction de Samuel Bianchini. Montrouge / Nancy : Burozoïque / Les Éditions du Parc et L'École nationale supérieure d'art de Nancy.

Bisenius-Penin (Carole) (sous la direction de). 2015. Résidence d'auteurs, création littéraire et médiations culturelles (1). À la recherche d'une cartographie. Nancy : PUN-Éditions universitaires de Lorraine.

Bordeaux (Marie-Christine) \& Caillet (Elisabeth). 2013. "La médiation culturelle : pratiques et enjeux théoriques ". Culture \& Musées. Hors-série. La muséologie 20 ans de recherches, p.139-161.

Cardon (Dominique). 2006. "La trajectoire des innovations ascendantes : université, coproduction et collectifs sur Internet » in Innovations, Usages, Réseaux / sous la direction de Chantal Charnet. Publication en ligne :<https://edutice.archives-ouvertes.fr/edutice-00134904/document>. 
Centre national des arts plastiques. 2016.223 Résidences d'arts visuels en France. Paris : Centre national des arts plastiques.

Chaumier (Serge). 2018. "Musées et patrimoine. Nouvelles formes de médiation, nouveaux projets ». Observatoire des politiques culturelles, 51, p. 40-43.

Couchot (Edmond) \& Hillaire (Norbert). 2003. L'Art numérique. Paris : Flammarion.

Dallet (Jean-Marie). 2012. "Une brève histoire des rencontres entre artistes, ingénieurs et scientifiques", p. 34-35 in Art \& culture(s) numérique(s) : panorama international / sous la direction de Dominique Roland. Enghien-les-Bains : Centre des arts d'Enghien-les-Bains.

Davallon (Jean). 2003. "La médiation : la communication en procès ?", MEI, 19, p. 39-59.

De Vrièse (Muriel), Martin (Bénédicte), Melin (Corinne), Moureau (Nathalie) \& Sagot-Duvauroux (Dominique). (2011). " Diffusion et valorisation de l'art actuel». Culture études, 1, p. 1-16.

During (Elie), Jeanpierre (Laurent) \& Kihm (Christophe) (sous la direction de). 2009. In actu. De l'expérimental dans l'art. Annecy / Dijon : Publications des Marquisats, École supérieure d'art de l'agglomération d'Annecy / Les Presses du réel.

Flichy (Patrice). 2010. Le Sacre de l'amateur. Sociologie des passions ordinaires à l'ère numérique. Paris : Seuil.

Fourmentraux (Jean-Paul). 2012. "Artiste, chercheur, innovateur : Le consortium Hexagram (Montréal)». Culture \& Musées, 19, p. 25-42.

Fourmentraux (Jean-Paul). 2011. Artistes de laboratoire, Recherche et création à l'ère numérique. Paris: Hermann.

Gardiès (Cécile) \& Fabre (Isabelle). 2012. «Définition et enjeux de la médiation numérique documentaire », p. 45-58 in Développer la médiation documentaire numérique / sous la direction de Xavier Galaup. Villeurbanne : Presses de l'ENSSIB.

Jeanneret (Yves). 2009. "La relation entre médiation et usages dans les recherches en information-communication en France ». RECIIS, 3, p. 25-34.

Le Falher (Olivier). 2014. «L'organisation du soutien public à l'expérimentation artistique : vers un modèle discontinu de consécration ". Communication \& management, 11, p. 83-94.

Lenoble (Catherine). 2011. "L'Europe des médias Labs. Cartographie des lieux et des réseaux ».MCD Media Labs in Europe, 62, p. 5-9.

Liquète (Vincent), Fabre (Isabelle) \& Gardiès (Cécile). 2010. " Faut-il reconsidérer la médiation documentaire? ". Les Enjeux de l'information et de la communication, 2, p.43-57. 
Menger (Pierre-Michel). 2009. Le Travail créateur. S'accomplir dans l'incertain. Paris : Seuil.

Ministère de la Culture et de la Communication. 2006. "Circulaire no 2006/001 du 13 janvier 2006 relative au soutien à des artistes et à des équipes artistiques dans le cadre de résidences", p. 5-9 in Bulletin officiel/ sous la direction de Martine Marigeaud. Paris : ministère de la Culture et de la Communication.

Théobalt (Jean-Christophe). 2001. "Le programme Espaces Culture Multimédia (ECM) ».Agora débats/jeunesses, 26, p. 79-85. 


\section{Auteur}

Stéphane Bellin, Avignon Université, Centre Norbert Elias, Équipe Culture \& Communication

Depuis juin 2017, Stéphane Bellin est titulaire d'un doctorat en communication de l'université d'Avignon et de pays de Vaucluse, ainsi que d'un doctorat en muséologie, médiation, patrimoine de l'Université du Québec à Montréal. Il est affilié à l'Équipe culture et communication du Centre Norbert Elias. Ses travaux portent sur les arts numériques, la communication de la recherche en art et plus largement la documentation et les archives dans le secteur des arts et de la culture. En 2014, il a publié un article intitulé "Documenter et communiquer la recherche en arts numériques par la mise en situation dialogale de l'artiste contemporain " dans Les Cabiers d'études supérieures en muséologie.

Courriel : stephane.bellin@netcourrier.com 


\section{La résidence confrontée à la recherche en arts numériques}

La recherche en arts numériques et ses acteurs investissent le modèle de la résidence artistique comme dispositif organisationnel. L'auteur analyse la façon dont celui-ci tente de prendre en compte la démultiplication des enjeux de production et de communication de cette discipline. Les attentes en matière de production se caractérisent par le retrait de l'œuvre au profit de l'expérimentation artistique et technologique. De même, les actions de médiation organisées ne montrent plus aux publics une ouvre finalisée, mais leur communiquent le travail artistique expérimenté et plus largement ses résultats fragmentés, tandis que la médiation documentaire assure une communication différée dans le temps. Il en ressort un modèle de résidence d'expérimentation et de recherche en arts numériques, pour lequel l'impératif de produire une ouvre est amoindri et pour lequel la fonction communicationnelle des actions de médiation demeure un axe fort.

\section{Mots-clés}

Résidence - arts numériques - recherche - médiation - médiation documentaire

\section{The residence facing research in digital arts}

Digital arts research and its actors invest the artistic residence model as an organizational device. The author analyses how the latter attempts to take into account the multiplication of production and communication issues in this discipline. Production expectations are characterized by the withdrawal of the work in favour of artistic and technological experimentation. Likewise, organised mediation actions no longer show audiences a finalised work, but communicate to them experienced artistic work and more widely its fragmented results, while documentary mediation ensures communication delayed in time. The result is a model of residency for experimentation and research in digital arts for which the imperative of producing a work is reduced and for which the communicative function of mediation actions remains a strong axis.

Keywords

Residence - digital arts - research - mediation - documentary mediation

\section{La residencia frente a la investigación en artes digitales.}

La investigación en artes digitales y sus actores, emplean el modelo de la residencia artística como un dispositivo organizativo. El autor analiza cómo, dicho modelo, intenta tomar en cuenta la multiplicación de 
desafíos de la producción y comunicación de esta disciplina. Las expectativas en la producción se caracterizan por el retiro de la obra en beneficio de la experimentación artística y tecnológica. Igualmente, las acciones de mediación organizadas ya no muestran al público un trabajo finalizado, sino que le comunican el trabajo artístico experimentado y más ampliamente sus resultados fragmentados, mientras que la mediación documental garantiza una comunicación diferida a lo largo del tiempo. El resultado es un modelo de residencia experimental y de investigación en artes digitales, cuyo imperativo de producir una obra es reducido, y para el cual, la función comunicacional de las acciones de mediación, sigue siendo un eje importante.

\section{Palabras clave}

Residencia - artes digitales - investigación - mediación - mediación documental 